\title{
Retrospective Analysis of Bladder Cancer Morphology and Depth of Invasion Under Cystoscopy
}

Hu chen

Yunnan Cancer Hospital, The Third Affiliated Hospital of Kunming Medical University Yang hong (D2447519483@qq.com)

Yunnan Cancer Hospital, The Third Affiliated Hospital of Kunming Medical University

Bai yu

Yunnan Cancer Hospital, The Third Affiliated Hospital of Kunming Medical University

Li ruiqian

Yunnan Cancer Hospital, The Third Affiliated Hospital of Kunming Medical University Li Jun

Yunnan Cancer Hospital, The Third Affiliated Hospital of Kunming Medical University

\section{Wu hongyi}

Yunnan Cancer Hospital, The Third Affiliated Hospital of Kunming Medical University

\section{Wang ziyong}

Yunnan Cancer Hospital, The Third Affiliated Hospital of Kunming Medical University

Jiang Haiyang

Yunnan Cancer Hospital, The Third Affiliated Hospital of Kunming Medical University

\section{Zhang Chongjian}

Yunnan Cancer Hospital, The Third Affiliated Hospital of Kunming Medical University

Bi ying

Yunnan Cancer Hospital, The Third Affiliated Hospital of Kunming Medical University

Wang qilin

Yunnan Cancer Hospital, The Third Affiliated Hospital of Kunming Medical University

\section{Research Article}

Keywords: Bladder cancer, invasive depth, morphology cystoscopy

Posted Date: September 27th, 2021

DOI: https://doi.org/10.21203/rs.3.rs-863072/v1 
License: (c) (i) This work is licensed under a Creative Commons Attribution 4.0 International License. Read Full License

Version of Record: A version of this preprint was published at BMC Urology on January 31st, 2022. See the published version at https://doi.org/10.1186/s12894-022-00958-0. 


\section{Abstract}

Background: The pathological diagnosis of bladder cancer workup relies on cystoscopy, however, due to sampling restriction, the depth of local invasion is often understaged.

Methods: A total of 386 patients with bladder urothelial carcinoma underwent follow-up. The data collected included age, sex, tumor size, surgical options, histologic grade, invasive depth, lymph node metastasis, and oncological outcomes, and the patients were divided into coral-like and crumb-like groups. These data were analyzed with the chi-square test, binary logistic regression, Kaplan-Meier analysis, univariable and multivariable logistic regression and Spearman correlation test.

Results: Bladder tumor morphology was moderately correlated with invasion depth $(\rho=0.492, p<0.001$; Spearman correlation), which was associated with invasion status (HR $=8.27 ; 95 \% \mathrm{Cl}: 4.3-15.79$, $\mathrm{p}$ <0.001). Tumor morphology was not an independent factor for OS but was associated with PFS. Outer invasion depth was an independent factor that was significantly associated with inferior OS and PFS.

Conclusions: Tumor morphology (coral-like and crumb-like) under cystoscopy was related to the depth of invasion. The outer invasive depth of BC was an independent factor that was significantly associated with inferior OS and PFS.

\section{Introduction}

Bladder cancer $(\mathrm{BC})$ is the $10^{\text {th }}$ most common cancer worldwide, and the second most common among urologic cancer, with approximately over 540,000 new cases and 200,000 deaths per year[1].

Tumors isolated to non-muscle-invasive bladder cancer (NMIBC) and muscle-invasive bladder cancer (MIBC)[2]. The accurate local staging of bladder carcinoma is key, as it has significant prognostic implications and determines treatment options. Non-muscle-invasive bladder carcinomas (Ta-T1) are suitable for localized treatment, either TURBT or intravesical chemotherapy, while radical cystectomy with LN dissection, remains the gold standard for muscle-invasive disease $(\geq T 2)[3,4]$.

The traditional diagnostic workup relies on cystoscopy and transurethral resection of bladder tumor (TURBT) to confirm the pathological diagnosis and muscle invasive status[3]; however, there is a discrepancy between preoperative pathological staging by cystoscopy biopsy and the postoperative pathologic staging based on surgery, with an inaccuracy rate of $23-50 \%$, mainly due to sampling error, particularly if there is an absence of the muscle layer in the specimen, which would understage the depth of local invasion[[5].

In addition, up to $25 \%$ of T1 tumors are eventually muscle invasive on subsequent TURBT, which entirely changes therapeutic management $[6,7]$.

Magnetic resonance imaging (MRI) can effectively cope with such limitations in local staging, and it is increasingly used for the preoperative, due to its high sensitivity to soft-tissue and ability to assess the 
depth of bladder wall invasion, with a recent meta-analysis reporting a high diagnostic performance in differentiating NMIBC from MIBC[8, 9].

In order to more accurately distinguish the muscular invasion state of bladder cancer, the VI-RADS score was developed in 2018. Multiparametric MRI (mpMRI), which incorporates morphological T2-weighted imaging (T2WI) alongside the functional sequences of diffusion-weighted imaging (DWI) and dynamic contrast-enhanced (DCE) imaging, has been shown to further improve the accuracy of primary and recurrent tumor detection and local staging $[9,10]$.

The sensitivity and specificity of a VI-RADS score of 3 or greater were $87.1 \%(95 \% \mathrm{Cl}$ : $78 \%-93 \%)$ and 96.5\% (95\% Cl: 93\%-98\%), respectively[11].

Cystoscopy is a simple, low-cost routine preoperative operation. Compared with MRI, it can observe the tumor size, position, shape, and surface nourishing blood vessels more clearly and intuitively under direct vision. At present, there are few literature summaries and analyses of tumors with different morphologies and depths of invasion under cystoscopy.

We hope to assess the correlation between the morphology of the tumor and the depth of invasion ,combined the morphology under cystoscopy with multiparametric mp-MRI to improve the accuracy of local T staging.

\section{Materials And Methods:}

Electronic medical records were reviewed to identify men treated for bladder cancer at Yunnan Cancer Hospital in China between December 2014 and May 2021. The study protocol was approved by the Yunnan Cancer Hospital Ethics Committee. Among 491 consecutive bladder cancer patients who were treated with surgery, 386 had sufficient follow-up data.

\section{Entry criteria:}

1 Tumors were pathologically diagnosed as urothelial carcinoma;

2 Cystoscopy was completed in our hospital before surgery;

3 The muscle tissue was observed in postoperative pathology.

\section{Exclusion criteria:}

1 Distant metastasis (M1);

2 Wide base or erosion or ulcer type or urethral mass of tumor;

3 Blurred vision during cystoscopy;

4 Tumors smaller than $1 \mathrm{~cm}$. 
According to Fig. 1, the 386 patients were divided into a coral-like group and a clump-like group based on the tumor inner circle radius ( $r$ ) and outer branch length (I) prior to surgery under cystoscopy.

Epidemiological and clinical data, including sex, age, tumor size, surgical options, histologic grade, tumor invasive depth, invasive status, lymph node metastasis status and oncological outcomes (OS and PFS), were collected. Survival analysis was performed by the Kaplan-Meier method for univariable analysis and the Cox regression method for multivariable analysis. The primary endpoints were OS and PFS, which were defined as the times from the date of pathological diagnosis to the dates of death and tumor progression, respectively. The Spearman correlation method was used to evaluate the correlation between the different morphology groups and invasive depths. Univariable (Kaplan-Meier) analysis was performed to determine any differences between the coral-like group and the clump-like group. Multivariable (Cox logistic regression) analysis was used to verify the independence of associations identified in univariable analyses and p-values.

\section{Results:}

The mean age $( \pm S D)$ of the patients was $61.37 \pm 11.9$ years. The patient data and their relationships to tumor morphology are summarized in Table 1.

The chi-square test showed that the coral-like group and clump-like group had significant differences in surgical methods, histologic grade, depth of invasion, and lymph node metastasis.

The Spearman correlation test showed that bladder tumor morphology was moderately correlated with invasion depth $(\rho=0.492, p<0.001)$ and invasion status $(\rho=0.467, p<0.001)$, whereas muscle-invasive status $(\rho=0.36, p<0.001)$, surgical methods $(\rho=0.213, p<0.001)$, histologic grade $(\rho=0.321, p<0.001)$, and lymph node metastasis $(\rho=0.167, p=0.001)$ were weakly correlated, and the remaining variables were not correlated (Table 2).

We used binary logistic regression to analyze the hazard ratios between invasive status and various factors, and the results suggest that tumor morphology was associated with invasive status $(H R=8.27$; $95 \% \mathrm{Cl}: 4.3-15.79, \mathrm{p}<0.001)$, partial cystectomy $(\mathrm{HR}=10.65 ; 95 \% \mathrm{Cl}: 2.74-41.37, \mathrm{p}=0.001)$ and radical cystectomy (HR $=15.75 ; 95 \% \mathrm{Cl}: 7.93-33.98, \mathrm{p}<0.001)$, as shown in Table 3.

Among the 386 patients, 371 survived (median follow-up duration 45 months, interquartile range 29-60 months). In total, 350 patients did not experience recurrence (median follow-up duration 43 months, interquartile range 22-58 months). Disease progression occurred in 36 patients, 20 of whom survived.

Kaplan-Meier analysis produced the following results: different bladder tumor morphologies (coral-like and clump-like) were not associated with OS, and tumor morphology was not associated with OS (logrank $p=0.206$ ) or PFS (log-rank $p=0.250)$, as shown in Fig. 2 .

In univariable analyses, tumor morphology was not associated with OS (HR $=1.9 ; 95 \% \mathrm{Cl}: 0.69-5.26, \mathrm{p}<$ 0.214). However, coral-like morphology was associated with better PFS (HR $=0.63 ; 95 \%$ Cl: 029-1.39, $p=$ 
0.225). In terms of the surgical type, radical cystectomy was significantly associated with inferior OS (HR $=16.49 ; 95 \% \mathrm{Cl}: 3.69-73.69, \mathrm{p}<0.001)$ compared to TURBT and not associated with PFS (HR $=1.48 ; 95 \%$ Cl: $0.73-2.99, p=0.274)$. Outer invasive depth was significantly associated with both inferior OS (HR= 78.49; 95\% Cl: 9.16-672.83, $\mathrm{p}<0.001)$ and PFS (HR $=4.57$; 95\% Cl: 1.7-12.33, $\mathrm{p}<0.002)$. Perivesical invasive depth was associated with inferior OS ( $\mathrm{HR}=34.27$; 95\% Cl: $3.83-306.93, \mathrm{p}=0.002)$ but was not associated with PFS (HR $=1.93 ; 95 \% \mathrm{Cl}: 0.65-5.7, \mathrm{p}<0.235)$. Lymph node metastasis status was significantly associated with inferior $\mathrm{OS}(\mathrm{HR}=13.46 ; 95 \% \mathrm{Cl}: 4.27-42.45, \mathrm{p}<0.001)$ and PFS $(\mathrm{HR}=5.25$; $95 \% \mathrm{Cl}: 1.86-14.88, \mathrm{p}=0.005)$.

We performed multivariate analysis, which revealed that tumor morphology was not a significant independent factor for OS ( $p=0.337)$ but was associated with PFS $(p=0.026)$. Outer invasive depth was an independent factor that was significantly associated with both inferior OS (HR $=53.74 ; 95 \% \mathrm{Cl}$ : 3.24892.21, $\mathrm{p}=0.005)$ and PFS (HR $=7.73 ; 95 \% \mathrm{Cl}: 2.06-29.06, \mathrm{p}<0.002)$. In the multivariate analysis, lymph node metastasis status remained significant for OS (HR $=6.36 ; 95 \% \mathrm{Cl}: 1.33-30.44, \mathrm{p}=0.021$ ) and PFS $(\mathrm{HR}=8.07 ; 95 \% \mathrm{Cl}: 1.9-34.27, \mathrm{p}=0.005)$, as indicated in the NCCN and EAU guidelines. However, age, sex, tumor size, surgical type and histologic grade were not significant for OS and PFS in the multivariable analysis (Table 4).

\section{Discussion}

The diagnosis of bladder cancer requires cystoscopy to obtain the tumor tissue for pathological diagnosis as the gold standard. The pathological results can provide clear information to distinguish between benign and malignant disease and different cancer types. However, it is difficult to obtain samples from the base of the tumor, resulting in inconsistent invasive depth of the preoperative and postoperative pathological diagnoses. The depth of infiltration of the preoperative bladder tissue is often understaged[5].

Preoperatively judging whether the tumor is noninvasive and invades the lamina propria can help the surgeon adjust the depth and breadth of the resection during the operation, maximize the resection to avoid recurrence of the tumor, and improve the prognosis. Second, whether there is muscle invasion directly determines the surgical type as TURBT or radical cystectomy. At present, judging whether there is muscle infiltration is usually determined by MRI. If MRI can be combined with the morphology of the tumor under cystoscopy to improve the accuracy of determining muscle infiltration status, a more reasonable surgical strategy can be chosen to benefit patients.

The relationship between tumor morphology and infiltration was analyzed through Cox regression. The risk of coral-like tumor invasion was 8.27 times that of crumb-like tumor invasion. Therefore, tumor morphology has important value for determining whether infiltration is present, and this information helps in the preoperative staging of tumors and in the selection of surgical type to benefit patients.

Univariate Cox regression indicated that morphology was mainly related to infiltration and had a relationship with the prognostic factors for OS and PFS. The results of multivariate analysis indicated 
that patients with crumb-like tumors had a longer PFS, which does not match our hypothesis. This may be because there are fewer TURBT options for patients with crumb-like tumors, radical resection or partial resection are other options, and crumb-like tumors are generally subjectively cut deeper during resection. These factors may be related to longer PFS. Second, infiltration reaching the deep muscle layer is a threshold and is associated with worse OS and PFS.

In the next step, MRI will be combined to establish a predictive model for preoperative invasion depth, hoping to improve the accuracy of judging the status and depth of invasion, which will help in the selection of surgical procedures and the prognosis of patients.

\section{Conclusions}

Tumor morphology (coral-like and crumb-like) under cystoscopy was related to the depth of invasion. The outer invasive depth of BC was an independent factor that was significantly associated with inferior OS and PFS.

\section{List Of Abbreviations}

$\mathrm{BC}$

Bladder cancer

TURBT

transurethral resection of a bladder tumor.

OS

overall survival

PFS

progression free survival

\section{Declarations}

\section{Ethics approval and consent to participate}

The study protocol was approved by the Yunnan Cancer Hospital Ethics Committee.All methods were carried out in accordance with relevant guidelines and regulations.Informed consent was obtained from all subjects.

\section{Consent for publication}

Not applicable

\section{Availability of data and materials}

The datasets used and analysed during the current study available from the corresponding author on reasonable request. 


\section{Competing interests}

The authors declare that they have no competing interests

\section{Funding}

The present study was supported by Applied Basic Research Foundation of Yunnan Province (no.202001AY070001-077),

National Cancer center Climbing Fund

(no.202001AY070001-077), National Natural Science Foundation of China(no.8216110765).Funds were used for purchasing the materials and had no role in the design, collection, analysis, or interpretation of data or writing of this manuscript.

\section{Authors' contributions}

$\mathrm{CH}, \mathrm{HY}$ were participated in the conception and initial design. YB, RQL,JL performed the clinical treatment of the bladder cancer, $\mathrm{CH}, \mathrm{HY}$ was a major contributor in writing the manuscript. $\mathrm{CH}, \mathrm{HYW}, \mathrm{ZYW}, \mathrm{HYJ}, \mathrm{CJZ}$ were participate in articles search, review, data extract, statistical analysis. YB, QLW were participate in project development and pictures processing. $\mathrm{CH}, \mathrm{YB}$ were participating in manuscript writing and revising. All authors have read and approved the manuscript.

\section{Acknowledgments}

Not applicable

\section{Conflict of Interest Disclosure}

There are no financial conflicts of interest to disclose

\section{References}

1. Bray F, Ferlay J, Soerjomataram I, et al. Global cancer statistics 2018: GLOBOCAN estimates of incidence and mortality worldwide for 36 cancers in 185 countries. 2018;68(6):394-424.

2. Lenis AT, Lec PM, Chamie KJJ. Bladder Cancer. JAMA. 2020;324(19):2006-.

3. Babjuk M, Böhle A, Burger M, et al. EAU guidelines on non-muscle-invasive urothelial carcinoma of the bladder: update 2016. 2017;71(3):447-61.

4. Witjes JA, Lebret T, Compérat EM, et al. Updated 2016 EAU guidelines on muscle-invasive and metastatic bladder cancer. 2017;71(3):462-75.

5. Caglic I, Panebianco V, Vargas HA, et al. MRI of bladder cancer: local and nodal staging. 2020;52(3):649-67. 
6. Gordon PC, Thomas F, Noon AP, et al. Long-term outcomes from re-resection for high-risk nonmuscle-invasive bladder cancer: a potential to rationalize use. 2019;5(4):650-7.

7. Thomas F, Noon AP, Rubin N, et al. Comparative outcomes of primary, recurrent, and progressive high-risk non-muscle-invasive bladder cancer. 2013;63(1):145-54.

8. Gandhi N, Krishna S, Booth CM, et al. Diagnostic accuracy of magnetic resonance imaging for tumour staging of bladder cancer: systematic review and meta-analysis. 2018;122(5):744-53.

9. Woo S, Suh CH, Kim SY, et al. Diagnostic performance of MRI for prediction of muscle-invasiveness of bladder cancer: a systematic review and meta-analysis. 2017;95:46-55.

10. Nguyen HT, Mortazavi A, Pohar KS, et al. Quantitative assessment of heterogeneity in bladder tumor MRI diffusivity: can response be predicted prior to neoadjuvant chemotherapy? 2017;3(4):237-44.

11. Wang H, Luo C, Zhang F, et al. Multiparametric MRI for bladder cancer: validation of VI-RADS for the detection of detrusor muscle invasion. 2019;291(3):668-74.

\section{Tables}


Table 1

Patients' characteristics

\begin{tabular}{|c|c|c|c|c|}
\hline & Total & Coral-like & Crumb-like & P-value \\
\hline $\operatorname{Sex}(n)$ & 386 & 264 & 122 & \\
\hline male & 299(78) & 204(77) & $95(78)$ & 0.896 \\
\hline female & $87(22)$ & $60(23)$ & $27(22)$ & \\
\hline Age (y) & $61.37 \pm 11.9$ & $60.09 \pm 12.57$ & $64.04 \pm 10.07$ & 0.526 \\
\hline \multicolumn{5}{|l|}{ Size (n) } \\
\hline$<3 \mathrm{~cm}$ & $271(70)$ & 190(72) & $81(66)$ & \multirow[t]{2}{*}{0.265} \\
\hline$>3 \mathrm{~cm}$ & $115(30)$ & $74(28)$ & $41(34)$ & \\
\hline \multicolumn{5}{|c|}{ Surgical options (n[\%]) } \\
\hline TURBT & $263(68)$ & 198(75) & $65(53)$ & \multirow[t]{3}{*}{$<0.001$} \\
\hline partial cystectomy & $21(5)$ & $11(4)$ & $10(8)$ & \\
\hline radical cystectomy & 102(26) & $55(20)$ & $47(38)$ & \\
\hline \multicolumn{5}{|c|}{ Histologic grade (n[\%]) } \\
\hline PUNLMP & $18(5)$ & $17(6)$ & $1(1)$ & \multirow[t]{3}{*}{$<0.001$} \\
\hline low grade & $105(27)$ & $94(35)$ & $11(9)$ & \\
\hline high grade & 263(68) & 153(58) & 110(90) & \\
\hline \multicolumn{5}{|l|}{ Invasive depth } \\
\hline non-muscle & $221(57)$ & 193(73) & $28(23)$ & \multirow[t]{5}{*}{$<0.001$} \\
\hline lamina propria & $87(23)$ & $44(17)$ & $43(35)$ & \\
\hline inner half & $33(8)$ & $14(5)$ & $19(16)$ & \\
\hline outer half & $16(4)$ & $8(3)$ & $8(6)$ & \\
\hline perivesical & $29(8)$ & $5(2)$ & $24(20)$ & \\
\hline \multicolumn{5}{|l|}{ Invasive status } \\
\hline no & $221(57)$ & 193(73) & $28(23)$ & \multirow[t]{2}{*}{$<0.001$} \\
\hline yes & 165(43) & $71(27)$ & $94(77)$ & \\
\hline \multicolumn{5}{|c|}{ Muscle-invasive status } \\
\hline no & $307(80)$ & 236(89) & $71(58)$ & $<0.001$ \\
\hline
\end{tabular}




\begin{tabular}{|c|c|c|c|c|}
\hline & Total & Coral-like & Crumb-like & P-value \\
\hline yes & $79(20)$ & $28(11)$ & $51(41)$ & \\
\hline \multicolumn{5}{|c|}{ Lymph node metastasis } \\
\hline no & $374(97)$ & 261(98) & 113(93) & \multirow[t]{2}{*}{0.001} \\
\hline yes & $12(3)$ & $3(1)$ & $9(7)$ & \\
\hline \multicolumn{5}{|c|}{ Recurrence (n[\%]) } \\
\hline no & $350(91)$ & 236(89) & 114(93) & \multirow[t]{2}{*}{0.203} \\
\hline yes & $36(9)$ & $28(11)$ & $8(7)$ & \\
\hline \multicolumn{5}{|c|}{ Vital status (n[\%]) } \\
\hline alive & $371(96)$ & $256(97)$ & 115(94) & \multirow[t]{2}{*}{0.2} \\
\hline dead & $15(4)$ & $8(3)$ & $7(6)$ & \\
\hline
\end{tabular}

Table 2

Correlation of bladder tumor morphology

\begin{tabular}{|lll|}
\hline Variables & Bladder tumor morphology & \\
\hline & Spearman correlation & P-value \\
\hline Sex (n[\%]) & -0.007 & 0.897 \\
\hline Age (y) & 0.14 & 0.006 \\
\hline Size (n[\%]) & 0.057 & 0.267 \\
\hline Surgical type (n[\%]) & 0.213 & $<0.001$ \\
\hline Histologic grade (n[\%]) & 0.321 & $<0.001$ \\
\hline Invasive depth & 0.492 & $<0.001$ \\
\hline Invasive status & 0.467 & $<0.001$ \\
\hline Muscle-invasive status & 0.36 & $<0.001$ \\
\hline Lymph node metastasis & 0.167 & 0.001 \\
\hline Recurrence (n[\%]) & -0.065 & 0.204 \\
\hline Vital status (n[\%]) & 0.065 & 0.202 \\
\hline
\end{tabular}


Table 3

Logistic regression of invasive status and other factors

\begin{tabular}{|c|c|c|c|}
\hline \multirow[t]{2}{*}{ Variables } & \multicolumn{3}{|c|}{ Invasive status } \\
\hline & HR & $95 \% \mathrm{Cl}$ & $\mathrm{p}$ \\
\hline \multicolumn{4}{|l|}{ Tumor morphology } \\
\hline coral-like & 8.27 & $4.3-15.79$ & $<0.001$ \\
\hline \multicolumn{4}{|l|}{ crumb-like } \\
\hline \multicolumn{4}{|l|}{ Size } \\
\hline$<3 \mathrm{~cm}$ & 1.30 & $0.66-2.59$ & 0.447 \\
\hline \multicolumn{4}{|l|}{$>3 \mathrm{~cm}$} \\
\hline \multicolumn{4}{|l|}{ Surgical type } \\
\hline TURBT & reference & - & - \\
\hline partial cystectomy & 10.65 & $2.74-41.37$ & 0.001 \\
\hline radical cystectomy & 15.75 & $7.93-33.98$ & $<0.001$ \\
\hline \multicolumn{4}{|l|}{ Histologic grade } \\
\hline PUNLMP & reference & - & - \\
\hline Low grade & 0.69 & $0.08-6.38$ & 0.692 \\
\hline High grade & 5.73 & $0.69-46.96$ & 0.104 \\
\hline
\end{tabular}

Due to technical limitations, table 4 is only available as a download in the Supplemental Files section.

Figures 


\section{coral-like $(I>r)$}
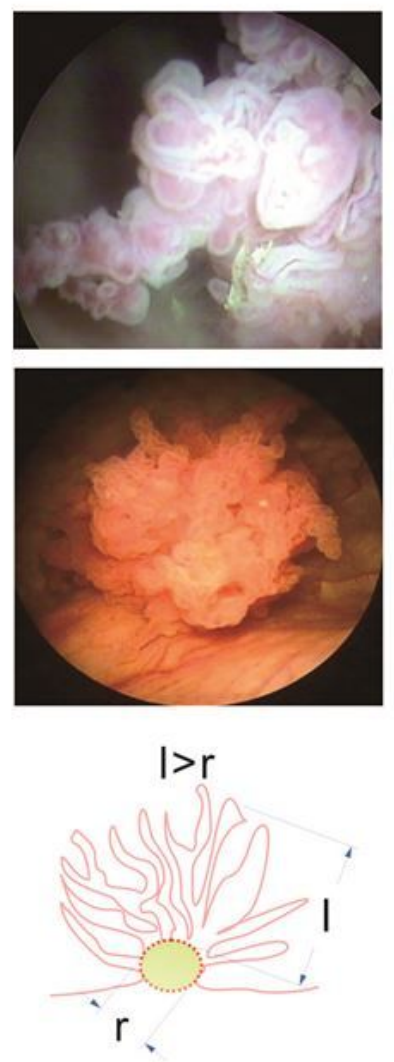
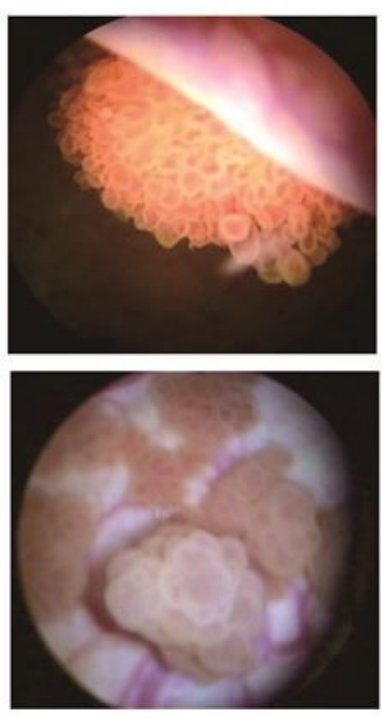

$1>r$

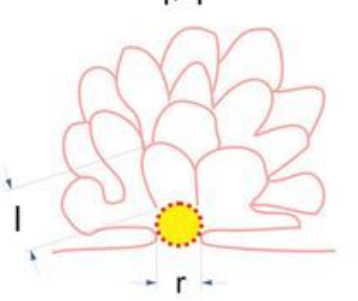

\section{crumb-like \\ $(r>I)$}
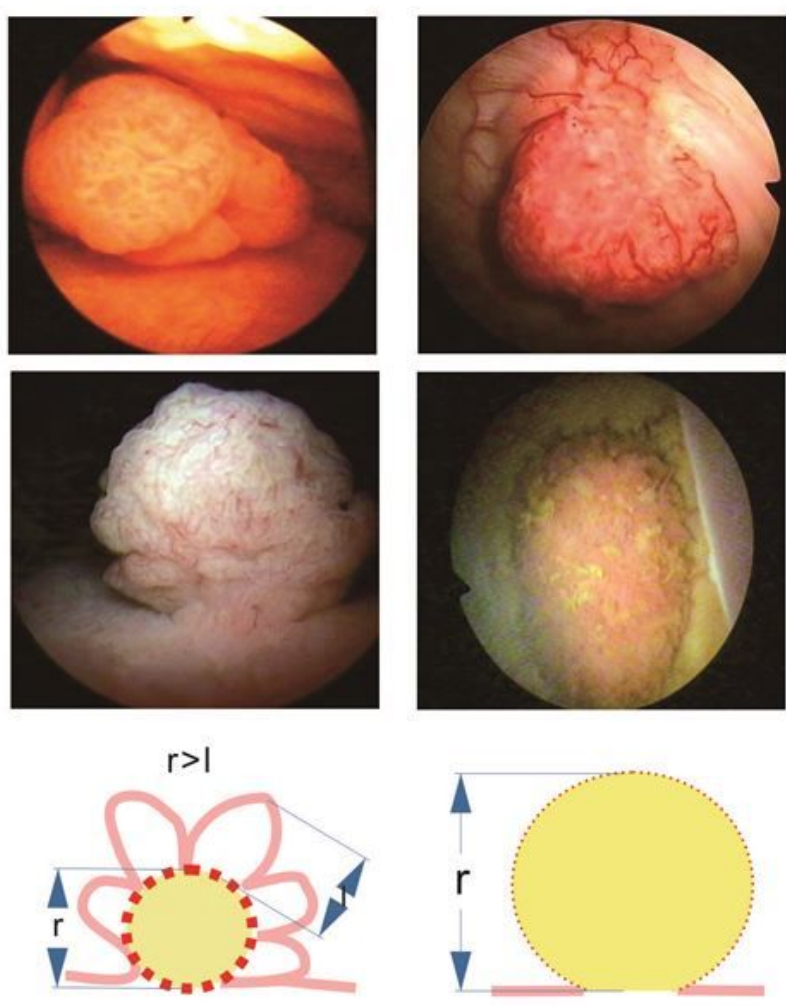

\section{Figure 1}

Division into two groups based on the tumor inner circle radius $(r)$ and outer branch length $(I)$ determined by preoperative cystoscopy. 


\section{Overall survival $(n=386)$}

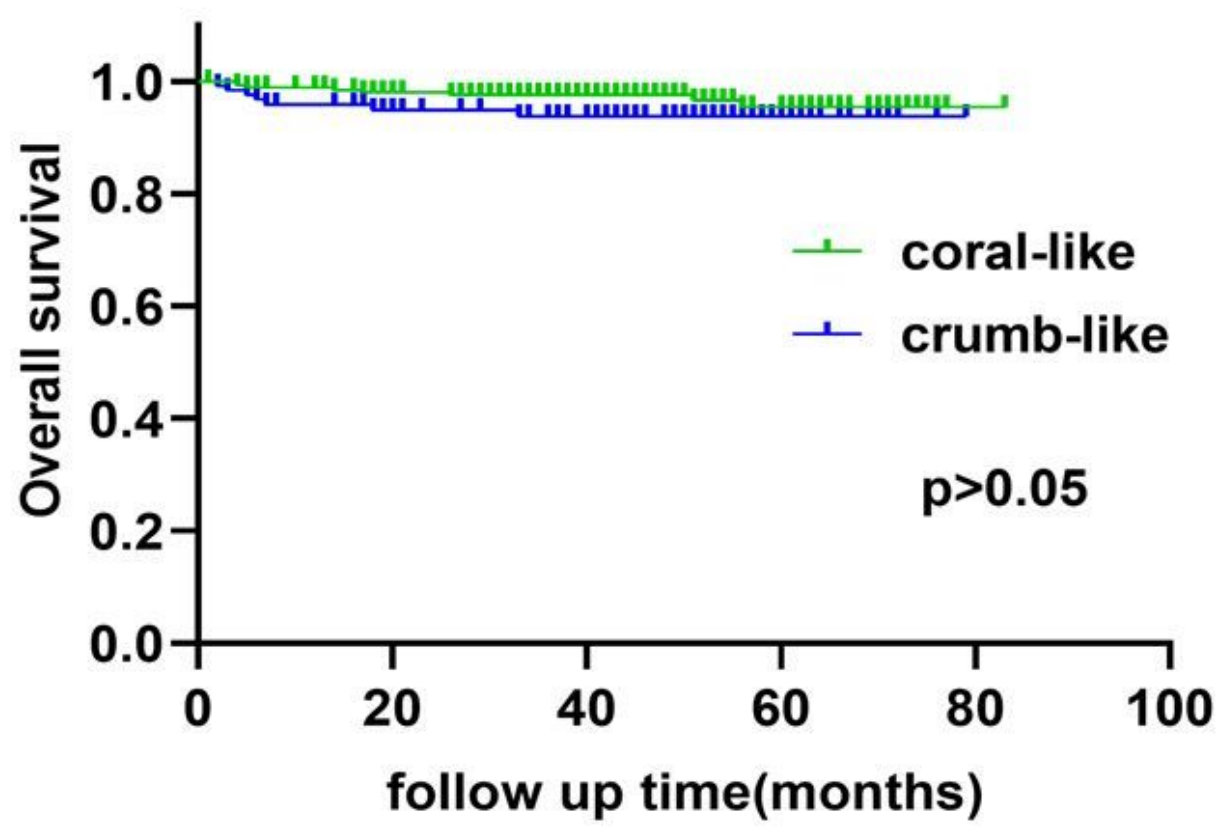

Progression free survival $(n=386)$

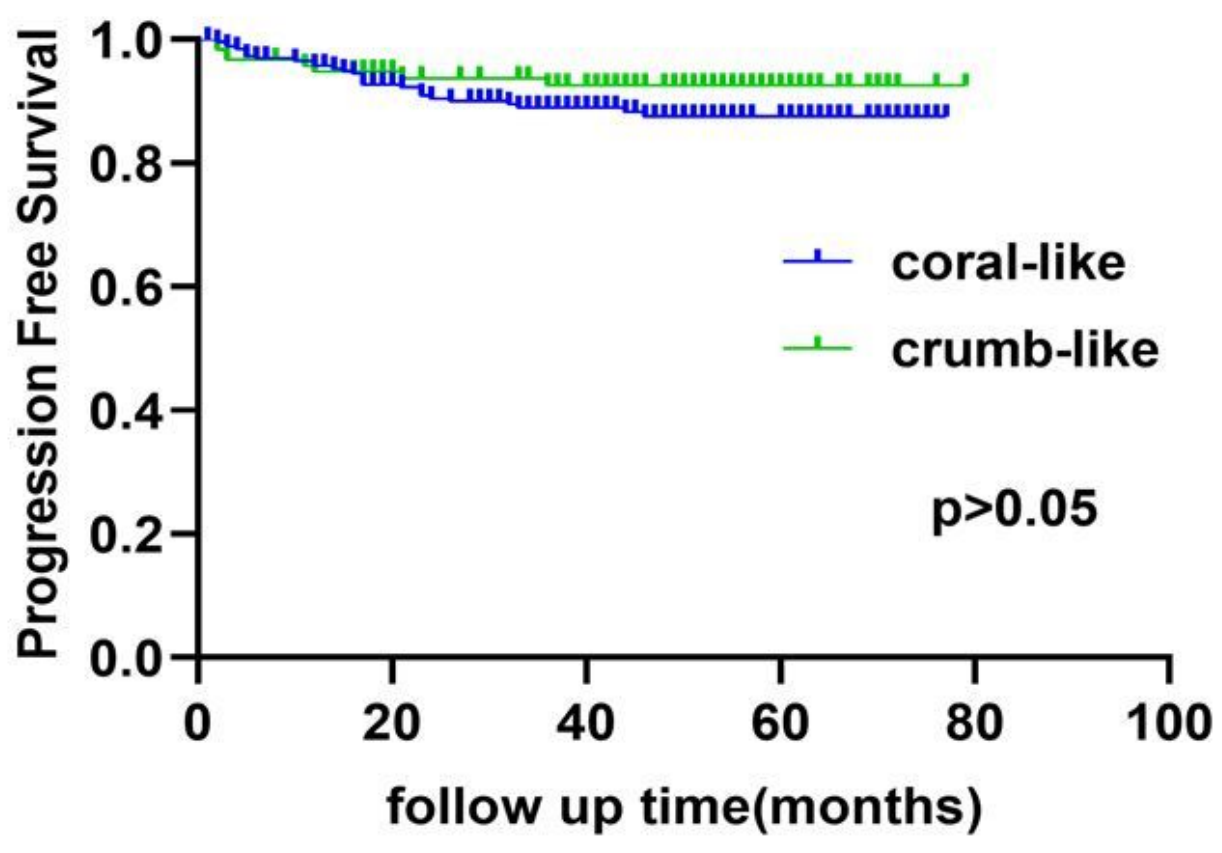

Figure 2

Follow up of OS and PFS

\section{Supplementary Files}

This is a list of supplementary files associated with this preprint. Click to download. 
- cystoscopymorphology.doc

Page 15/15 